\title{
Análise da equação de Swamee-Jain para cálculo do fator de atrito
}

\author{
Leandro Andrade ${ }^{1} \&$ Jacinto de A. Carvalho ${ }^{2}$
}

\author{
1 Irrigação e Drenagem/UFLA. Rua Irlanda, BI. B1, Apto. 403, Residencial Paineiras, Parque das Nações, CEP 74593-250, \\ Goiânia, GO. E-mail: leandro@pivotequip.com.br (Foto) \\ 2 Departamento de Engenharia/UFLA, Lavras, MG. Fone: (35) 3829-1489. E-mail: jacintoc@ufla.br
}

Protocolo $156-21 / 12 / 2000$

\begin{abstract}
Resumo: Objetivou-se, com este trabalho, estudar o comportamento da equação de Swamee-Jain nas mais variadas situações de condução de água em sistemas pressurizados, para isto se desenvolveu um aplicativo computacional para realizar simulações variando o diâmetro interno do tubo, a velocidade de escoamento e a rugosidade absoluta das paredes da tubulação. A base de comparação do estudo se compunha das equações mais empregadas no cálculo do fator de atrito da equação universal de Darcy-Weissbach, respeitando-se seus respectivos regimes de escoamento e limitações. A partir de então, concluiu-se que, para o regime turbulento em conduto liso, a equação de Swamee-Jain apresentou diferenças de valores de $\mathrm{f}$ em relação às equações específicas requerendo, para melhor precisão, um fator de correção. Para os outros tipos de escoamento a concordância foi considerada satisfatória.
\end{abstract}

Palavras-chave: hidráulica, perda de carga, irrigação

\section{Analysis of the Swamee-Jain's equation for the calculation of friction factor}

\begin{abstract}
The aim of this research was to study the behavior of the equation of Swamee-Jain in the most varied situations of conduction of water in pressurized systems. A software was developed to accomplish simulations varying the internal diameter of the pipe, mean velocity of flow and absolute roughness of the pipes. The base of comparison of the study was the most employed equations in the calculation of the of friction factor of the universal equation of Darcy-Weissbach, respecting the respective drainage regimes and limitations. For the turbulent regime in flat conduit, it was observed that the equation of Swamee-Jain presented differences of values of $f$ in relation to the specific equations, needing, for a better precision, a correction factor. For the other drainage types the agreement was considered satisfactory.
\end{abstract}

Key words: hydraulic, loss of load, irrigation

\section{INTRODUÇÃO}

O sucesso de implantação da agricultura irrigada depende da otimização do projeto hidráulico, buscando sempre a menor soma dos custos fixos e variáveis. Neste caso, um dos parâmetros mais importantes é a perda de carga, a qual deve ser determinada com precisão, resultando no sistema de recalque mais econômico. Darcy e Weissbach apresentaram uma expressão geral de perda de carga válida para qualquer líquido e regime de escoamento em condutos forçados (Azevedo Neto et al., 1998). Entre as variáveis empregadas nesta fórmula, o fator de atrito - fé o parâmetro de mais difícil determinação (Macintyre, 1987; Vianna, 1997; Azevedo Neto et al., 1998).

Os regimes de escoamento são classificados em função do número de Reynolds: laminar (inferior a 2000) e turbulento (acima de 4000). O regime turbulento é sub-dividido em outros três tipos: condutos lisos, turbulento de transição e turbulência plena. Em cada um, fé determinado por equações empíricas que só podem ser empregadas no regime de escoamento em que foram ensaiadas (Neves, 1989).

De acordo com Azevedo Neto (1998) e Carvalho (2000) as expressões de Blasius (Eq. 1), von Kárman-Prandtl (Eq. 2) e Nikuradse (Eq. 3) são recomendadas no regime turbulento em condutos lisos, as equações de Colebrook-White (Eq. 4), Moody (Eq. 5) e Prandtl-Colebrook (Eq. 6) no regime turbulento de transição e para o regime de turbulência plena se utiliza a equação de Nikuradse (Eq. 7):

$$
\begin{gathered}
f=0,316(\operatorname{Re})^{-0,25} \\
\frac{1}{\sqrt{f}}=2 \log (\operatorname{Re} \sqrt{f})-0,8
\end{gathered}
$$




$$
\begin{gathered}
\mathrm{f}=0,0032+0,221(\mathrm{Re})^{-0,237} \\
\frac{1}{\sqrt{\mathrm{f}}}=-2 \log \left(\frac{2 \times \varepsilon}{3,71 \times \mathrm{D}}+\frac{2,51}{\operatorname{Re} \times \sqrt{\mathrm{f}}}\right) \\
\mathrm{f}=0,0055\left[1+\left(20.000 \frac{\varepsilon}{\mathrm{D}}+\frac{10^{6}}{\mathrm{Re}}\right)^{\frac{1}{3}}\right] \\
\frac{1}{\sqrt{\mathrm{f}}}=1,74-2 \log \left(\frac{2 \varepsilon}{\mathrm{D}}+\frac{18,7}{\operatorname{Re} \times \sqrt{\mathrm{f}}}\right) \\
\frac{1}{\sqrt{\mathrm{f}}}=1,74-2 \log \left(\frac{2 \varepsilon}{\mathrm{D}}\right)
\end{gathered}
$$

em que:

$$
\begin{array}{ll}
\text { Re } & \text { - número de Reynolds, adimensional } \\
\varepsilon & \text { - rugosidade absoluta do tubo, } \mathrm{mm} \\
\mathrm{D} & \text { - diâmetro interno do tubo, } \mathrm{mm}
\end{array}
$$

Entretanto, Swamee \& Jain (1976), citados por Porto (1998), apresentaram uma expressão geral (Eq. 8) que calcula o fator de atrito (f) sem restrições quanto ao regime de escoamento, número de Reynolds e rugosidade relativa.

$$
\mathrm{f}=\left\{\left(\frac{64}{\operatorname{Re}}\right)^{8}+9,5 \times\left[\operatorname{Ln}\left(\frac{\varepsilon}{3,7 \times \mathrm{D}}+\frac{5,74}{\mathrm{Re}^{0.9}}\right)-\left(\frac{2500}{\mathrm{Re}}\right)^{6}\right]^{-16}\right\}^{0,125}
$$

Apesar da expressão de Swamee-Jain ser de aplicação geral, os projetistas ainda relutam em não utilizá-la, devido ao número reduzido de estudos sobre esta equação, podendo ocorrer desvios capazes de condenar um sistema de irrigação, seja pelo superdimensionamento, elevando os custos, seja pelo subdimensionamento, resultando em vazões e pressões inferiores às projetadas.

Partindo-se do princípio de que as expressões empíricas determinam satisfatoriamente o valor $\mathrm{f}$, realizou-se um estudo comparativo entre essas equações e a equação geral de SwameeJain, a fim de se comprovar o seu uso em qualquer situação de regime de escoamento citada.

\section{MATERIAL E MÉTODOS}

A equação geral de Swamee-Jain requer três variáveis: número de Reynolds (Re), rugosidade absoluta do material $(\varepsilon)$ e diâmetro do conduto (D). No presente estudo, variaram-se esses parâmetros, de forma a simular todas as situações prováveis de ocorrer em um sistema de recalque para irrigação.

Os valores de rugosidade absoluta do conduto empregados, foram 0,005, 0,05 e 0,5 mm. Essas rugosidades englobam os tubos construídos com materiais de PVC, aço laminado novo, aço comercial, aço zincado, ferro fundido novo e ferro fundido asfaltado, dentre outros (Azevedo Netto, 1998; Porto, 1998).

Uma vez que vários estudos indicam que a velocidade econômica em sistemas de bombeamento varia entre 0,7 e $2,5 \mathrm{~m} \mathrm{~s}^{-1}$, foram utilizadas, neste trabalho, velocidades entre 0,5 e 4,0 $\mathrm{m} \mathrm{s}^{-1}$, aumentando os limites recomendados, enquanto os diâmetros utilizados variaram entre 12 e $300 \mathrm{~mm}$. Combinando as velocidades de escoamento com valores de diâmetros, obtiveram-se valores do número de Reynolds variando entre 6.000 e 1.170 .000 . A rugosidade relativa $(\varepsilon / D)$ variou entre 0,00001667 e 0,0417 e a viscosidade cinemática utilizada foi de $0,000001 \mathrm{~m}^{2} \mathrm{~s}^{-1}$, sendo que este valor se refere à água com temperatura próximo de $20^{\circ} \mathrm{C}$.

Desenvolveu-se um aplicativo computacional em Visual Basic, visando simular as diversas situações citadas e aplicar, para cada situação, a expressão para cálculo de f recomendada e a equação geral de Swamee-Jain, cujos resultados foram exportados para as planilhas do Microsoft Excel, onde se calcularam os desvios (Eq. 9) da equação geral de SwameeJain, em relação a cada equação analisada, sendo que os menores desvios determinaram melhor desempenho da expressão em estudo.

$$
\Delta f(\%)=\frac{f s-\text { fo }}{\text { fo }} 100
$$

em que: fs - fator de atrito calculado pela equação geral de Swamee-
Jain, adimensional
fo - fator de atrito calculado pelas demais equações,
adimensional

\section{RESULTADOS E DISCUSSÃO}

O regime de escoamento turbulento em conduto liso ocorreu apenas nas rugosidades absolutas de 0,005 e 0,05 $\mathrm{mm}$ e as equações utilizadas como base de comparação foram as equações de Blasius, de von Kárman-Prandtl e de Nikuradse. Os resultados da média dos desvios estão apresentados na Tabela 1 e na Figura 1.

Tabela 1. Valores médios dos desvios apresentados pela equação de Swamee-Jain em relação às equações de Blasius, von Kárman-Prandtl e Nikuradse, no regime de escoamento turbulento em condutos lisos

\begin{tabular}{lcc}
\hline \multirow{2}{*}{ Equações } & \multicolumn{2}{c}{ Desvio (\%) } \\
\cline { 2 - 3 } & $\varepsilon=0,005$ & $\varepsilon=0,05$ \\
\hline Blasius & 1,9 & 13,9 \\
von Kárman-Prandtl & 2,7 & 14,3 \\
Nikuradse & 5,0 & 17,8 \\
\hline
\end{tabular}

Em todos os casos, as maiores variações de desvios ocorreram com os menores valores de número de Reynolds. Como os valores obtidos pelas expressões empíricas foram considerados corretos, a expressão estudada superestimou os valores neste regime de escoamento, requerendo a utilização de um fator de ajuste para a equação de Swamee-Jain. 


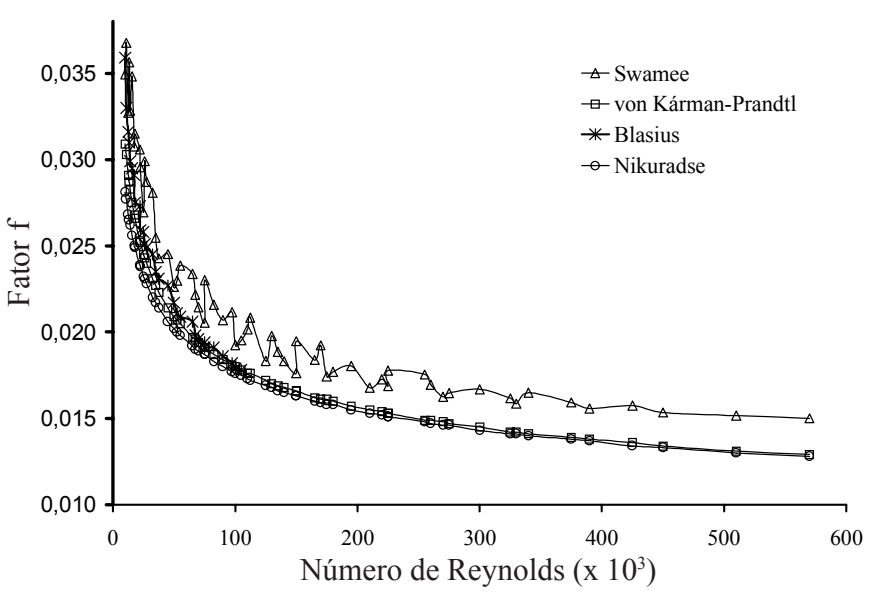

Figura 1. Comparação gráfica entre os valores de $f$ calculados pelas equações de Blasius, von Kárman-Prandtl e Nikuradse, do regime turbulento em condutos lisos e de Swamee-Jain, em função do número de Reynolds, para $\varepsilon=0,05 \mathrm{~mm}$

Os desvios calculados no regime de escoamento turbulento de transição (Tabela 2) representado pelas equações de Colebrook-White, Prandtl-Colebrook e Moody, aumentaram com a rugosidade absoluta e diminuíram com o aumento do número de Reynolds, porém, a equação geral de Swamee-Jain subestimou os valores do fator de atrito f em relação à equação de Colebrook-White, razão do sinal negativo dos desvios.

Tabela 2. Valores médios dos desvios apresentados pela equação de Swamee-Jain em relação às equações de Colebrook-White, Prandtl-Colebrook e Moody, no regime de escoamento turbulento de transição

\begin{tabular}{lcc}
\hline \multirow{2}{*}{ Equações } & \multicolumn{2}{c}{ Desvio (\%) } \\
\cline { 2 - 3 } & $\varepsilon=0,05$ & $\varepsilon=0,5$ \\
\hline Colebrook-White & $-11,7$ & $-18,6$ \\
Prandtl-Colebrook & 0,7 & 0,8 \\
Moody & $-0,5$ & 1,3 \\
\hline
\end{tabular}

Observa-se, pela Figura 2, a concordância entre as equações de Prandtl-Colebrook e Moody com Swamee-Jain, o que não acontece com relação à equação de Colebrook-White, a qual superestima o valor de f. Verifica-se, portanto, que os desvios apresentados pela equação em análise, neste caso, são menores que as diferenças encontradas entre as equações específicas.

Comparando-se os resultados obtidos da expressão estudada com os resultados da expressão de Nikuradse para regime turbulento em condutos rugosos, a média dos desvios foi de $1,8 \%$. Este regime de escoamento ocorreu apenas na rugosidade absoluta de $0,5 \mathrm{~mm}$ e os desvios também diminuíram com o aumento do número de Reynolds (Figura 3).

De forma geral, a equação geral de Swamee-Jain apresentou resultados satisfatórios para a maioria das situações analisadas, excetuando-se para o regime turbulento de conduto liso, casos em que, para melhor aproximação com os valores produzidos pelas equações específicas, seria necessária a introdução de um fator de correção. Entretanto, é importante considerar que a maioria dos condutos, utilizados em tubulações para condução de água, apresentam rugosidades maiores que $0,05 \mathrm{~mm}$ (Porto, 1998), e, os escoamentos, em sua maioria, ocorrem com maiores valores do número de Reynolds, o que conduz a escoamentos turbulento de transição ou de turbulência plena, condições em que a equação de Swamee-Jain apresentou bons resultados.

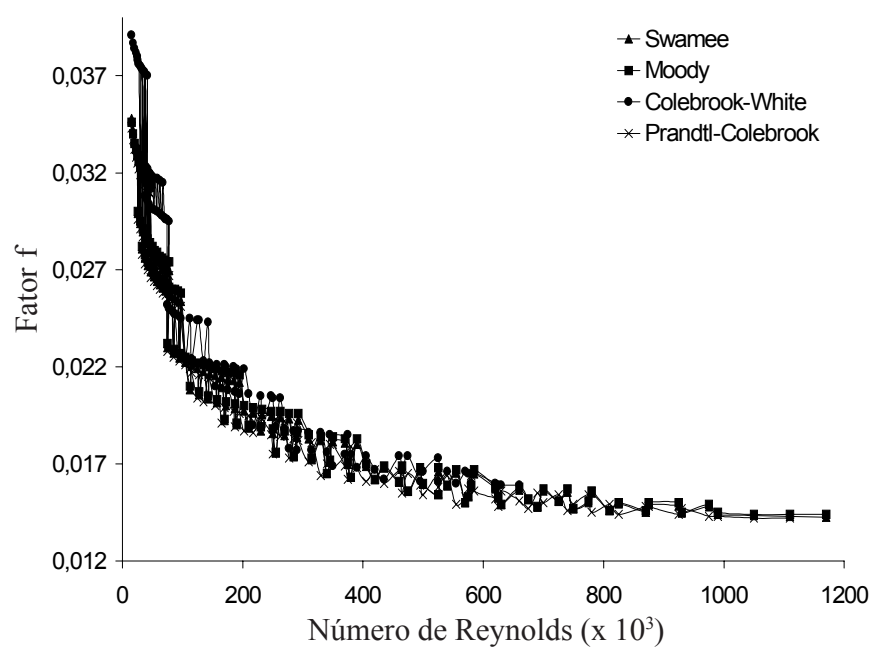

Figura 2. Comparação gráfica entre os valores de $\mathrm{f}$ calculados pelas equações Colebrook-White, Prandtl-Colebrook e de Moody do regime turbulento de transição e de Swamee-Jain, em função do número de Reynolds, para $\varepsilon=0,05 \mathrm{~mm}$

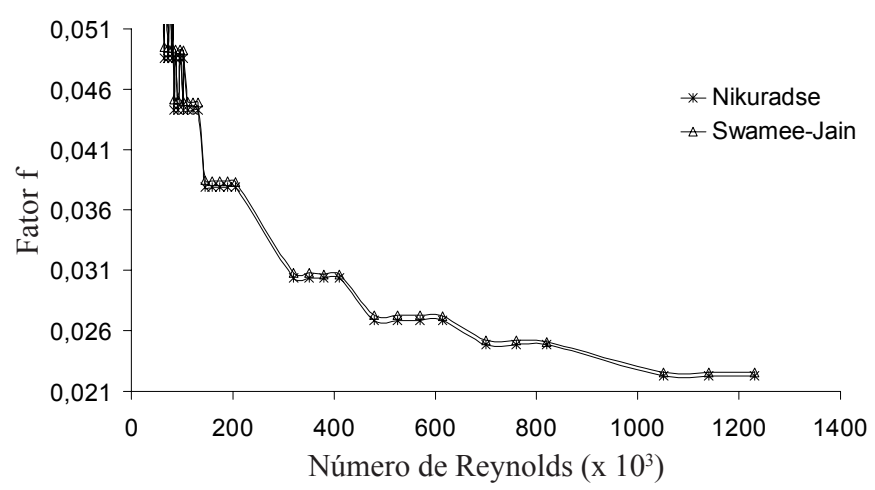

Figura 3. Comparação gráfica entre os valores de $f$ calculados pela equação de Nikuradse para o regime de turbulência plena, e de Swamee-Jain, em função do número de Reynolds, para $\varepsilon=0,5 \mathrm{~mm}$

A rugosidade absoluta $(\varepsilon)$ de difícil determinação prática e precisa, deveria ser fornecida pelo fabricante do conduto; entretanto, raramente se encontram informações técnicas a este respeito para a grande maioria dos tubos comerciais; além disso, os valores da rugosidade absoluta encontrados em bibliografias técnicas, são bastante variáveis apresentando, para um mesmo tipo de material, uma ampla faixa de valores, causando dúvidas e dificultando a tomada de decisão pelo técnico projetista.

Ante esta discussão, conclui-se que a equação de SwameeJain, apesar de apresentar, em algumas situações, diferenças de valores de f em relação às equações específicas, é bastante útil no dimensionamento de tubulações, pois o seu uso não exige a determinação, a priori, do tipo de regime de escoamento (pode ser utilizada para qualquer tipo de escoamento) e permite o cálculo do fator de atrito f sem necessidade de procedimento interativo.

\section{CONCLUSÕES}

1. Os desvios apresentados pela equação de Swamee-Jain, em relação às equações para o regime turbulento em conduto liso, aumentaram com a rugosidade absoluta do tubo e foram menores com o crescimento do número de Reynolds. 
2. Para o regime turbulento de transição e de turbulência plena, os valores de $\mathrm{f}$ apresentados pela equação de SwameeJain, foram bastante próximos àqueles dados pelas equações específicas, exceto para Colebrook-White.

3. A expressão estudada teve um bom ajuste na maioria dos casos, sendo o seu uso feito com cautela.

\section{LITERATURA CITADA}

Azevedo Netto, J.M.; Fernandez Y Fernandez, M.; Araujo, R. de; Ito, A.E. Manual dehidráulica. 8.ed. São Paulo: Blücher, 1998.669p.
Carvalho, J. de A. Mecânica dos fluidos e hidráulica. Lavras: UFLA. 2000.241p. Apostila

Macintyre, A.J. Bombas e instalações de bombeamento. 2.ed. Rio de Janeiro: Ed. Guanabara Dois. 1987. 782p.

Neves, E.T. Curso de hidráulica. 9.ed. São Paulo: Globo, 1989. $577 \mathrm{p}$.

Porto, R.M. Hidráulica básica. São Carlos: EESC-USP. 1998. 540p.

Vianna, M.R. Mecânica dos fluidos para engenheiros. 3 ed. Belo Horizonte: Imprimatur, 1997. 582p. 